\title{
THE RELATIONSHIP OF IDIOPATHIC CARDIAC HYPERTROPHY TO FOETAL ENDOCARDITIS
}

\author{
BY \\ L. E. GLYNN, M.D., M.R.C.P., and J. D. L. REINHOLD, M.A., B.M., M.R.C.P. \\ From the Unit for Juvenile Rheumatism, the Canadian Red Cross Memorial Hospital, \\ Taplow, Bucks.
}

(Received for Publication, August 11, 1949)

Idiopathic cardiac hypertrophy in children may be divided into those cases associated with glycogen infiltration, and those without. A characteristic feature of the latter is the presence of gross fibroelastic thickening of the endocardium (Kugel and Stoloff, 1933; Mahon, 1936). This thickened endocardium, frequently associated with myocardial scarring, has suggested that an inflammatory basis underlies the condition. Gross (1941), however, in a review of so-called foetal endocarditis, has brought convincing evidence against this concept, and attributes the myocardial changes to impaired nutrition secondary to the endocardial thickening.

The case presented here suggests that endocardial fibro-elastosis, which is found in $70 \%$ of cases of so-called foetal endocarditis (Gross, 1941) may, in cases surviving the neonatal period, lead to the condition of idiopathic cardiac hypertrophy in children, the myocardial hyperplasia being secondary to the endocardial thickening.

\section{Case Report}

P.D., a boy aged 21 months, was admitted to the Canadian Red Cross Memorial Hospital on Jan. 31, 1949, under the care of Dr. Reginald Lightwood. He died suddenly at the end of March, 1949, presumably from cardiac failure. He was an illegitimate child whose father was not known; the mother was healthy and there were no siblings. At birth, he was a normal baby weighing $7 \mathrm{lb}$.; he was breast fed for eight months and weaning was carried out normally. His early development was normal and he was walking at 15 months of age.

He had bronchitis and mild jaundice immediately after birth, and four attacks of bronchitis in the last year of his life. Fifteen months before he died he was in hospital with bronchopneumonia, and then was considered to be mentally backward. He had had no other illnesses.

One month before admission he started to cough, became fretful, and lost his appetite. Three days later he had a slight rise of temperature. This was unaffected by sulphonamides, but subsided while he was given oral penicillin. Two weeks later his face became puffy, and shortly afterwards his legs and hands also became swollen. On Jan. 28, three days before admission, his abdomen was enlarged and he was rather breathless. At this time he vomited occasionally after food.

On admission the baby was pale, apyrexial, orthopnoeic, and slightly cyanosed. There was considerable oedema of the face, hands, forearms, abdominal wall, legs, and sacral region, and ascites. Slightly enlarged glands were palpable in both axillae and in both posterior triangles of the neck. The throat was not inflamed. The apex beat was not palpable, and the area of cardiac dullness was increased both to the left and to the right. The heart sounds were muffled and there was a soft systolic murmur at the apex. There was no pericardial friction. The pulse was of low tension (125 per minute). The jugular venous pressure was raised $7 \mathrm{~cm}$. above the sternal angle at

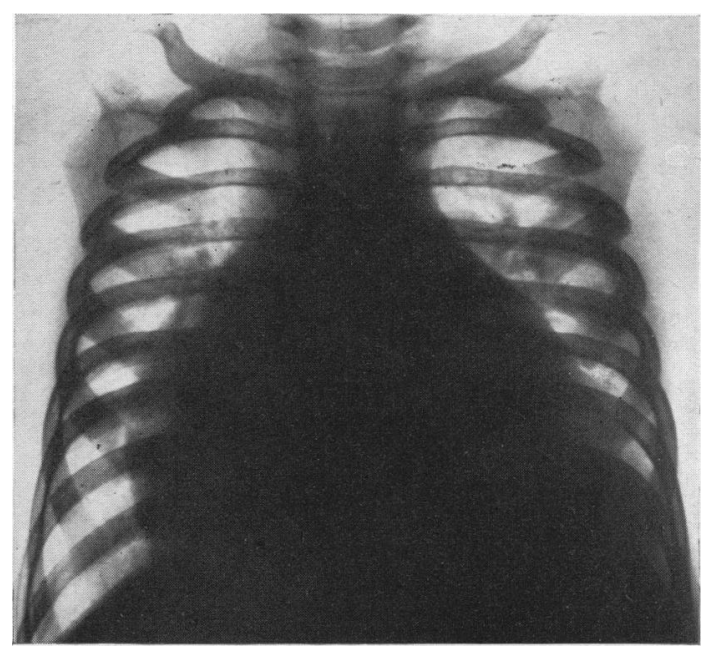

FIG. 1.-Radiograph of chest showing gross enlargement of the cardiac shadow. 
$45^{\circ}$, but no jugular pulsation was visible. The blood pressure was $90 / 65$. There were diffuse rhonchi in all areas over both lungs, and the respiration rate was 40 per minute; there was diminished air entry at the left base. The liver was enlarged $6 \mathrm{~cm}$. below the costal margin in the mid-clavicular line. The fundi were normal. A radiograph of the chest showed generalized enlargement of the cardiac shadow suggestive of pericardial effusion (Fig. 1); screening of the heart showed no evidence of cardiac pulsation except for a small area at the upper left border. The electrocardiogram showed normal standard leads and upright $T$ waves in leads $\mathrm{V} 1$ and $\mathrm{V} 2$, and the corrected Q-T interval was $0.410 \mathrm{sec}$.

(Fig. 2). The urine showed 45 mg. $\%$ albumin and a few epithelial cells ; subsequently there was no other abnormality and usually no albuminuria. The red blood count was 3.9 million with $11 \cdot 7$ g. haemoglobi n. Th e white blood count was 13,800 with $26 \%$ p o 1 y morphs, $68 \%$ 1 m mhocytes, $5 \%$ monocytes, and $1 \%$ e osin op hils. The serum protein level was 4 g. $\%$, and the albumin-globulin ratio $1: 1$. The serum urea level was $57 \mathrm{mg} . \%$ and the serum cholesterol level $500 \mathrm{mg} . \%$. The antistreptolysin titre was less than 50 units per ml. A tuberculin jelly and Mantoux test $(1: 1,000)$ were both negative.

Progress. A week after admission oedema was less, but the jugular venous pressure was still raised, and the liver was enlarged $6 \mathrm{~cm}$. Tachycardia of 100-130 persisted throughout the illness. During the first fortnight in hospital the baby lost $6 \mathrm{lb}$. in weight and the oedema almost completely disappeared, after which it became evident that there was generalized muscular wasting. The heart and liver did not alter materially in size. One month after admission he developed a respiratory infection which was treated with penicillin, and a week later generalized oedema recurred. An attempted aspiration of the pericardium was unsuccessful on three occasions, and the impression was gained that the pericardial sac was not thickened. Two months after admission fluoroscopy showed the heart shadow unaltered in size and without any obvious pulsation, but the appearances were believed to be compatible with gross cardiac dilatation. A tentative diagnosis of glycogenic cardiomegaly was made, and investigations for glycogen storage disease performed. The fasting blood sugar was $84 \mathrm{mg} . \%$, and 15 minutes after the injection of adrenaline the blood sugar was $78 \mathrm{mg}$. \%. The urine had a trace of acetone in it, but no sugar. The colloidal gold and thymol turbidity tests were both slightly positive. There was an improvement in the anaemia after the first two months of the illness, but leucocytosis persisted throughout. His appetite was good throughout the illness. The patient died suddenly, exactly three months after admis sion, while being was hed in bed.

\section{Post-mortem} Report

The subject was a male infant of average build and a fair state of nutrition, with no external evidence of developmental abnormality.

Heart. (Weight, 140 g.) The heart was considerably enlarged owing to hypertrophy of both ventricles, especially the left. The parietal pericardium was firmly adherent to the anterior surface of the right ventricle and to the right auricular appendage. There was no evidence of recent pericarditis. The pericardial fluid was clear and of normal volume. The endocardium of both ventricles was strikingly thickened and opaque and very conspicuous around the hypertrophied muscle trabeculae. The mitral and tricuspid valves showed unusual translucence, with possibly myxomatous nodules $1-3 \mathrm{~mm}$. in diameter projecting from the free edge of the cusps (Fig. 3). No rheumatic vegetations were seen. The aortic and pulmonary valves were normal. The right auricle was considerably dilated and the appendage filled with 


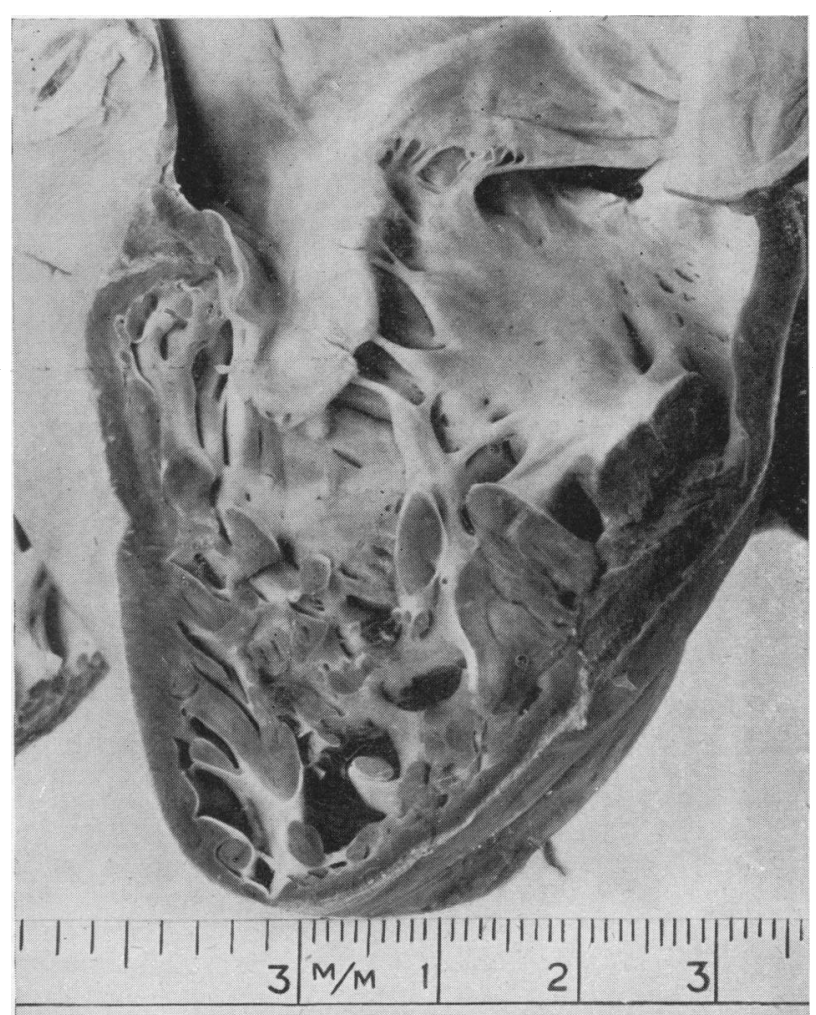

FIG. 3.-The heart opened to expose the right side of the interventricular septum and the tricuspid valve. Note the nodular thickening towards the free margin of the septal cusp, and the endocardial thickening, especially on the septum.

ante-mortem thrombus. The foramen ovale and the ductus arteriosus were both closed. The coronary vessels and the aorta were healthy.

Valve measurements:

Aortic, $40 \mathrm{~mm}$. Mitral, $58 \mathrm{~mm}$. Tricuspid, $72 \mathrm{~mm}$. Pulmonary, $54 \mathrm{~mm}$.

Thickness of ventricles:

Left ventricle, anterior, $9 \mathrm{~mm}$.; posterior, $10 \mathrm{~mm}$;

Right ventricle, anterior, $3.5 \mathrm{~mm}$.; posterior, $4.5 \mathrm{~mm}$.

Lungs. (Weight: right, 135 g.; left, 110 g.) There was about $50 \mathrm{ml}$. of slightly cloudy, yellow fluid in the left pleural cavity. Some old fibrous adhesions were found between the right lower lobe and the diaphragm. Both lungs were well expanded, but firmer than normal in consistency and slightly rusty brown in colour: There was moderate oedema in the right upper lobe but not elsewhere. Frothy fluid was found in small quantities in the trachea and bronchi. The hilar glands were not enlarged.

Liver. (Weight, 345 g.) This organ was a little above average size, pale yellowish brown, with the lobular markings accentuated. The sectioned surface showed the bright orange yellow colour of the periportal zones, contrasting with the greyish brown, slightly depressed centrilobular zones. The consistency was firmer than normal, and the organ cut with a distinctly fibrotic resistance. The gall bladder, bile ducts, and pancreas were normal.

The spleen (weight, 32 g.) was normal.

The kidneys, a little above average size, were pale but otherwise normal, as also were the pelves, ureters, bladder, and genitalia.

The thyroid, thymus, and adrenals were normal.

The alimentary canal was normal. A very considerable quantity of clear yellow fluid was found in the general peritoneal cavity.

The brain (weight, 960 g.) was normal.

Histological Examination. The following findings are recorded:

Mitral Valve and Left Ventricle. There was marked hypertrophy of the subendothelial trabeculae and considerable thickening and elastosis of the endocardium (Fig. 4), with no evidence of inflammation except in the pericardium. In some places encroachment into the myocardium had occurred with atrophy of the muscle fibres. There was no inflammation in the valve.

TRICUSPID Valve and Right Ventricle. There was no evidence of valvulitis, but considerable hypertrophy of the muscle trabeculae and thickening of their endocardium. The pericardium was chronically inflamed with moderate hyperaemia, lymphocyte and plasma cell infiltration. There was endocardial thickening, very rich in elastic fibres. Moderate sized branches of the coronary arteries showed intimal thickening with elastic reduplication.

TRICUSPID CUSP. There was myxomatous swelling

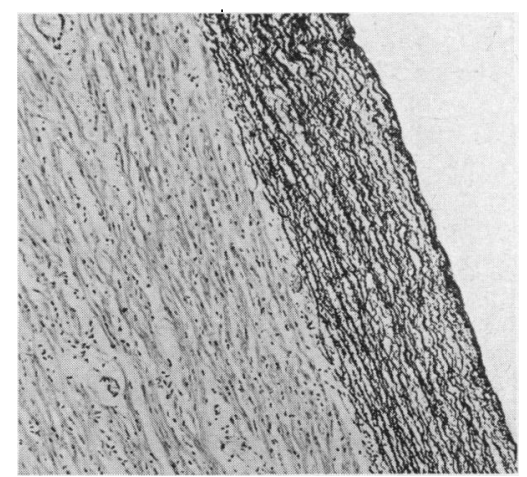

Frg. 4.-Section of left ventricle showing fibro-elastosis of the endocardium. (Weigert's elastic stain and neutral red. $\times 65$.) 
at free margin, but no evidence of inflammation (Fig. 5).

LEFT AURICLE. The endocardium was much

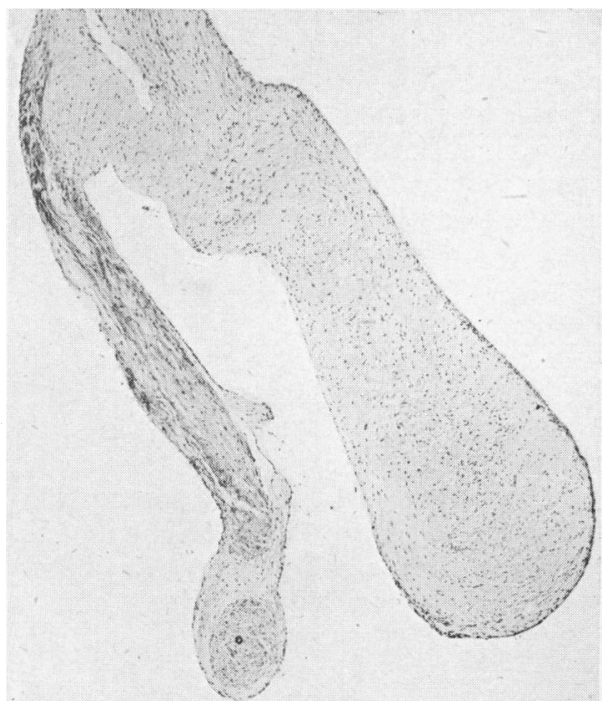

FIG. 5.-Section through a cusp of the tricuspid valve showing the myxomatous nodular thickening of the free margin. Note the absence of any inflammatory infiltration. (Haematoxylin and eosin. $\times 25$.)

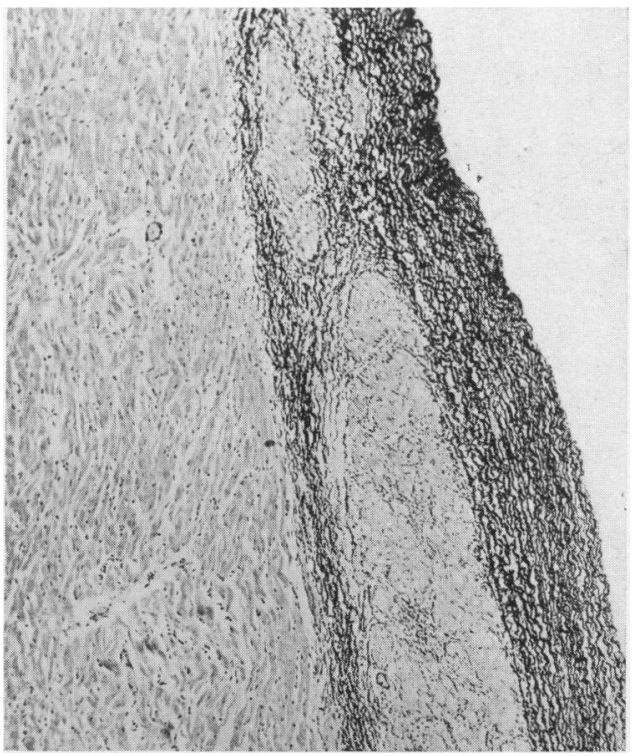

FIG. 6.-Section of the interventricular septum showing the great thickening of the endocardium, and the striking elastic hyperplasia. Embedded in this thickened endocardium are bundles of Purkinje-like fibres. (Weigert's elastic stain and neutral red. $\times 55$.) thickened with striking elastosis, and there was chronic inflammatory thickening of the pericardium. The visceral pericardium showed striking papillary proliferation near the attachment of the parietal pericardium.

RIGHT AURICLE. The endocardium was not thickened. The serous pericardium was thrown into papillary folds in the angle of reflexion onto the parietal layer.

Right Auricular Appendage. Extensive, adherent ante-mortem thrombus showing early organization was shown.

INTERVENTRICULAR SEPTUM. The endocardium on the right surface was much thickened, and contained bundles of vacuolated swollen cardiac muscle fibres in its middle layer (Fig. 6). A striking

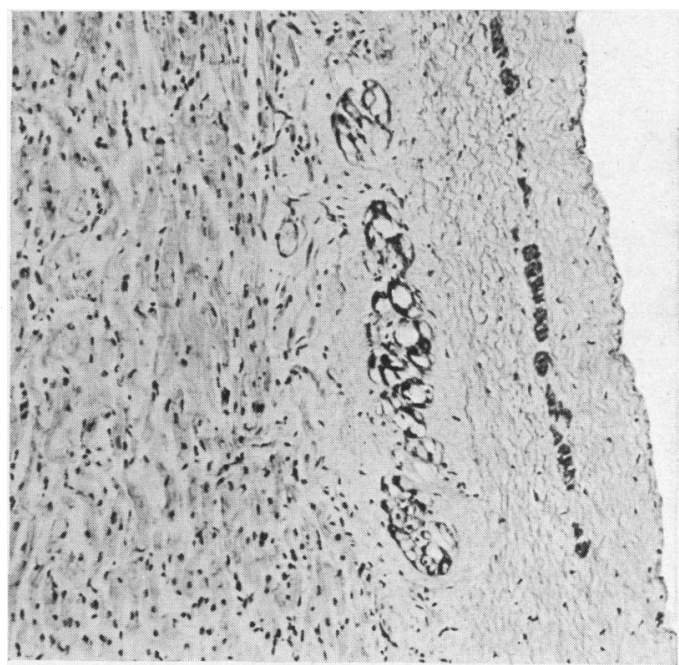

FIG. 7.-Thickened endocardium on the right surface of the interventricular septum. Bundles of fibres with central vacuole and ring of glycogen are seen in cross section in the deeper part of the endocardium. The ordinary myocardial fibres are not vacuolated. (Glycogen stain. $\times 90$.)

elastic hyperplasia in the superficial and to a less extent in the deep layer of the endocardium was seen. Coarse granules of polysaccharide, presumably glycogen, were conspicuous in the endocardial muscle fibres, but not elsewhere in the myocardium (Fig. 7).

AORTIC VALVE. The ground substance was myxomatous. There was no valvulitis.

Papillary Muscle. The endocardium was thickened with severe elastosis, especially towards the apex.

LIVER. The centrilobular zones showed passive congestion, hydropic and fatty degeneration and necrosis. The periportal zone cells were infiltrated with glycogen. The portal tracts were unusually conspicuous because of lymphocytic infiltration, 
bile duct proliferation, and fibrosis, especially in the left lobe. The capsular lymphatics were distended.

SPLEEN. Lymphoid hyperplasia and passive congestion were seen.

KIDNEY. Eosinophil reticular material was found in the capsular spaces, and several foetal type glomeruli.

\section{Discussion}

It is apparent that the hypertrophy in the heart in this case is not due to glycogen infiltration, as the muscle fibres lack the vacuolated appearance characteristic of that condition (Pompe, 1933; Finkelstein, 1936; Carrington and Korumbhaar 1924). Moreover, the staining for polysaccharide substances showed negligible amounts of glycogen in the myocardial fibres. This was not due to faulty technique, as the persisting Purkinje fibres gave a characteristic and quite heavy reaction for glycogen (Fig. 7). The positive adrenaline test was probably due to the fact that the adrenaline was not properly absorbed owing to the gross oedema; and the liver glycogen was consistent with the appearances usually seen in children in cases of sudden death (Bodian, personal communication).

A survey of the literature reveals quite clearly that endocardial elastosis is not a feature of glycogenic cardiomegaly (Finkelstein, 1936; van Creveld and van der Linde, 1939; Pompe, 1932; Antopol and others, 1934; Humphreys and Kato, 1934). In marked contrast are the findings in the nonglycogen group of cases of idiopathic cardiac hypertrophy, in which almost all those published have shown gross endocardial thickening (Kugel and Stoloff, 1933; Levine, 1934; Mahon, 1936; Weinberg and Himelfarb, 1943; Vulliamy, 1947).

In Gross's review of the literature on so-called foetal endocarditis, he mentions that $70 \%$ of cases show fibro-elastic thickening of the endocardium. As endocardial elastosis is rare, it is all the more noteworthy that it should occur in both these conditions, hitherto considered to be separate entities. This strongly suggests that idiopathic hypertrophy of the heart is a late result of so-called foetal endocarditis in cases surviving the neonatal period. On this hypothesis, idiopathic hypertrophy of the myocardium is probably a work hypertrophy resulting from interference with normal heart action. The precise mechanism is not clear, but the presence of a thick fibro-elastic endocardium must impede diastole and systole. In diastole, the elasticity of the thickened endocardium will impose a resistance to cardiac filling. In systole, it might appear at first sight that the elastic recoil would assist emptying of the heart; however, the systolic wave occurs from apex to base, and any superadded contraction out of step with the normal sequence must cause a resistance to cardiac outflow.
Despite the macroscopic finding of nodular thickening of the valves, no evidence of inflammation was found in our case, the thickening consisting merely of myxomatous tissue of embryonic appearance (Fig. 5). This, together with the absence of cellular infiltration in the endocardium, strongly supports Gross's contention that so-called foetal endocarditis is not of inflammatory origin. It also serves to differentiate the condition from Fiedler's myocarditis, in which there are acute inflammatory changes in the interstitial tissue of the heart. The occasional finding by other observers of lymphocytic infiltration in the myocardium (Kugel and Stoloff, 1933) can be explained by Gross's theory to be a result of myocardial damage due to nutritional interference by a thickened endocardium. Dissmann (1932) has suggested that the endocardial changes represent a primary developmental defect; the persistence in our case of Purkinje fibres within the thickened endocardium, indicative of the early stage at which the change takes place(Robb, Kaylor, and Truman, 1948), lends further support to this view.

\section{Summary}

The phenomenon of fibro-elastic hyperplasia of the endocardium in idiopathic cardiac hypertrophy and in so-called foetal endocarditis is discussed. A relationship between these two conditions is suggested largely on the basis of this common finding, and it is illustrated by a fatal case of endocardial fibro-elastosis with myocardial hypertrophy in a boy aged 21 months.

We are indebted to Dr. Paul Wood and Dr. Martin Bodian for their advice, and to Dr. Reginald Lightwood for permission to publish this case and for his helpful criticism.

\section{REFERENCES}

Antopol, W., Heilbrunn, J., and Tucman, L. R. (1934). Amer. J. med. Sci., 188, 354.

Bodian, M. Personal Communication.

Carrington, G. L., and Korumbhaar, E. B. (1924). Amer. J. Dis. Child., 27, 449.

Creveld, S. van, and van der Linde, H. M. (1939). Arch. Dis. Childh., 27, 14.

Dissmann, E. (1932). Frankfurt. Z. Path., 43, 476.

Finkelstein, L. E. (1936). Amer. J. med. Sci., 191, 415. Gross, P. (1941). Arch. Path., 31, 163.

Humphreys, E. M., and Kato, K. (1934). Amer, J. Path., 10, 589.

Kugel, M. Ä., and Stoloff, E. G. (1933). Amer. J. Dis. Child., 45, 828.

Levine, H. D. (1934). Amer. J. Dis. Child., 48, 1072.

Mahon, G. S. (1936). Amer. Heart J., 12, 608.

Pompe, J. C. (1933). Ann. Anat. path. med. chir., 10, 23. - (1932). Ned. Tijdschr. Geneesk., 76, 304.

Robb, J. S., Kaylor, C. T., and Turman, W. Q. (1948). Amer. J. Med., 5, 324.

Vulliamy, D. G. (1947). Brit. Heart J., 9, 161.

Weinberg, T., and Himelfarb, A. J. (1943). Bull. Johns Hopk. Hosp., 72, 299. 